\title{
La difesa dei territori dell'Ossola, sul corridoio spagnolo delle Fiandre, negli ultimi decenni del secolo XVII
}

The defense of Ossola territories, on the Spanish passageway to Flanders, in the late seventeenth century

\author{
Paolo Negri \\ Architect, Villadossola (VB), Italy, paolo.negri@libero.it
}

\begin{abstract}
The Ossola territories, in the area to the northwest of Milan, have constituted the western border most in contact with the nordic and tens-alpine world, ever since the first establishment of the Duchy of Milan. It is already known from G. Parker's monography on the camino español that one of the common routes, which allowed overland redeployment of Spanish troops headed towards Flanders, from the Liguria region across central Europe, would go through Ossola and cross the Simplon Pass or the Gries Pass. During the turbulent historical period of the Thirty Years' War and the following one, the changing fortunes of the Duchy of Milan in Spanish hands led to the fast and strategic conquest of Piedmontese cities (1639) and their equally rapid loss on the western border. Especially in the second half of the seventeenth century, the Franco-Savoy advance threw the Piedmontese borders into a severe crisis and the Spanish governors of Milan accordingly adopted all the military measures needed to address the issue. Fearing incursions from the north, through Romandie, Valais and Ossola, in the late seventeenth century, many field engineers among whom Beretta and Formenti, arranged the transformation of Domodossola, the outermost military stronghold only equipped with obsolete medieval walls at the time, into a "modern" rampart city (1687-1690). The engineers produced an accurate study of the territory, preserved today in the Historical Civic Archive and at the Trivulziana library in Milan in a cartographic manuscript series of all the Ossola valleys and the Swiss territory from Brig to Lake Leman.
\end{abstract}

Keywords: Ossola, Beretta, Formenti, spanish Flanders corridor.

\section{Introduzione}

Curiosa circostanza quella in cui si trovò il Marchese sabaudo Vincenzo Ferrero d'Ormea, governatore di Novara, durante la visita che effettuò in Ossola nell'autunno del 1768. A Domodossola trovò i cittadini più preoccupati delle loro vendemmie e dei loro raccolti che delle difese delle mura, con l'antica cortina e i fossati adibiti ad allegri giardini terrazzati di vigne e frutteti; scrive il governatore: "coi loro orti vanno fino contro lo stesso muro di cinta, in modo che non compare più nessuna vestigia di spalto", "anzi si vedono piante da frutta verdeggiare sotto le mura e persino pali delle viti infisse nelle mura". Qua e là case appoggiate alle fortificazioni, finestre aperte nelle mura, panni stesi sui rivellini o lungo le merlature. ${ }^{1}$ 


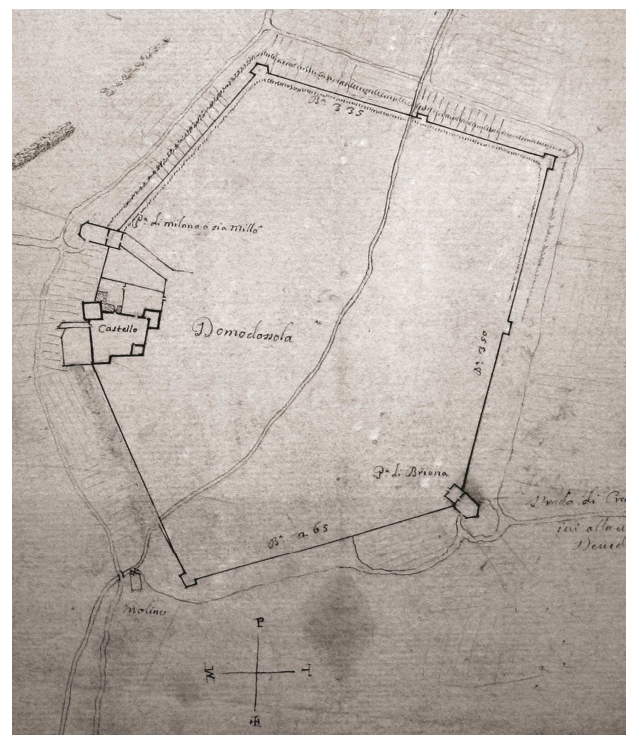

Fig. 1. (Carlo Giovan Battista) Formenti, disegno a penna, pianta orientata, sul verso "Domodossola /

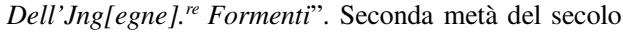
XVII. Particolare delle mura della città. [Inedito]. Archivio Storico Civico e Biblioteca Trivulziana (d'ora in poi BTMi), Belgioioso, 267.

Situazione piuttosto incresciosa considerando che circa ottant' anni prima i governatori spagnoli si erano ostinati a trasformare il borgo in una moderna cittadella fortificata, temendo un'invasione dei francesi, a seguito dell'imbarazzante incidente che causò, per le salve dell'artiglieria durante i festeggiamenti della liberazione di Vienna dall'assedio dei Turchi nel 1683, l'esplosione della Torre delle Polveri del castello del borgo, lasciandolo così sguarnito di difese (Sesti, 1707, p. 12; Bossi, 2017, p. 313). É pur anche vero che già nel gennaio del 1744 , dopo il passaggio dei territori del Novarese dalla Lombardia al Piemonte, stabilito nel Trattato di Worms, le munizioni rimanenti del castello di Domodossola furono trasportate nel castello di Milano $^{2}$ e le vestigia militari domesi adombrassero già la loro trasformazione in stazioni di sosta per le diligenze e in alberghi a favore dei nuovi viaggiatori di fine '700 e ' 800 , mutamento di destinazione favorito dal passaggio della route napoleonica del Sempione che, ad inizio secolo XIX, portò allo sventramento delle due porte urbiche (Negri, 2014).

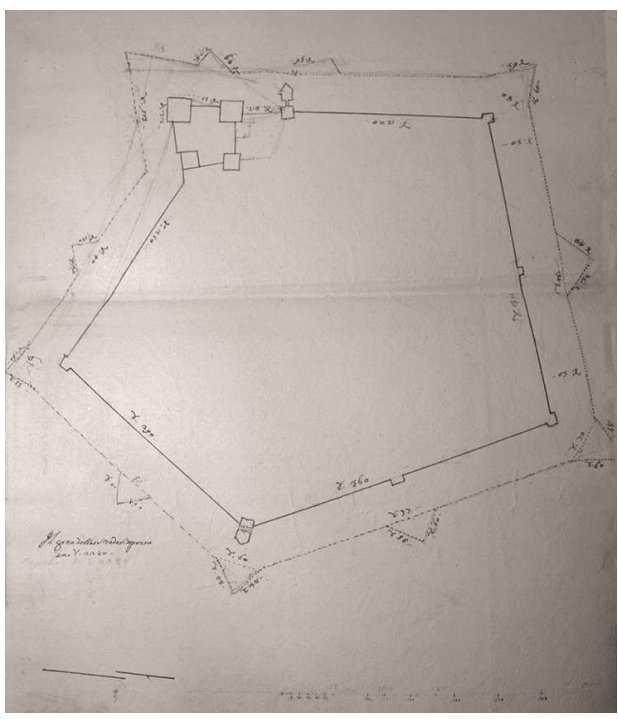

Fig. 2. Anonimo ma (Gaspare) Beretta, disegno a matita, in basso a sinistra Il giro della strada coperta. 1690. BTMi, Belgioioso, 267 (Inedito).

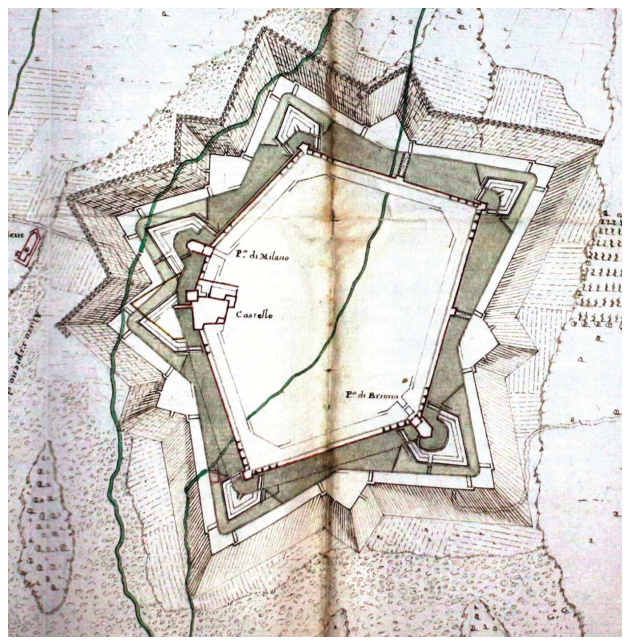

Fig. 3. Anonimo ma Gaspare (Beretta), Borgo di Domodossola, seconda metà del XVII secolo, disegno a penna acquerellato, paesaggio con parti in rilievo, Scala de braza di Milano 500, 713 x 472 mm, didascalie. Particolare del progetto di fortificazione alla moderna del borgo di Domodossola. BTMi, Belgioioso, 267 (Rotary Club Milano Aquileia, 1999; Rizzi, 2017, pp. 76-77).

\section{Le fortificazioni della città di Domodossola}

Ben prima, dopo l'ammodernamento del castello e della cinta muraria di Domodossola, compiuta 


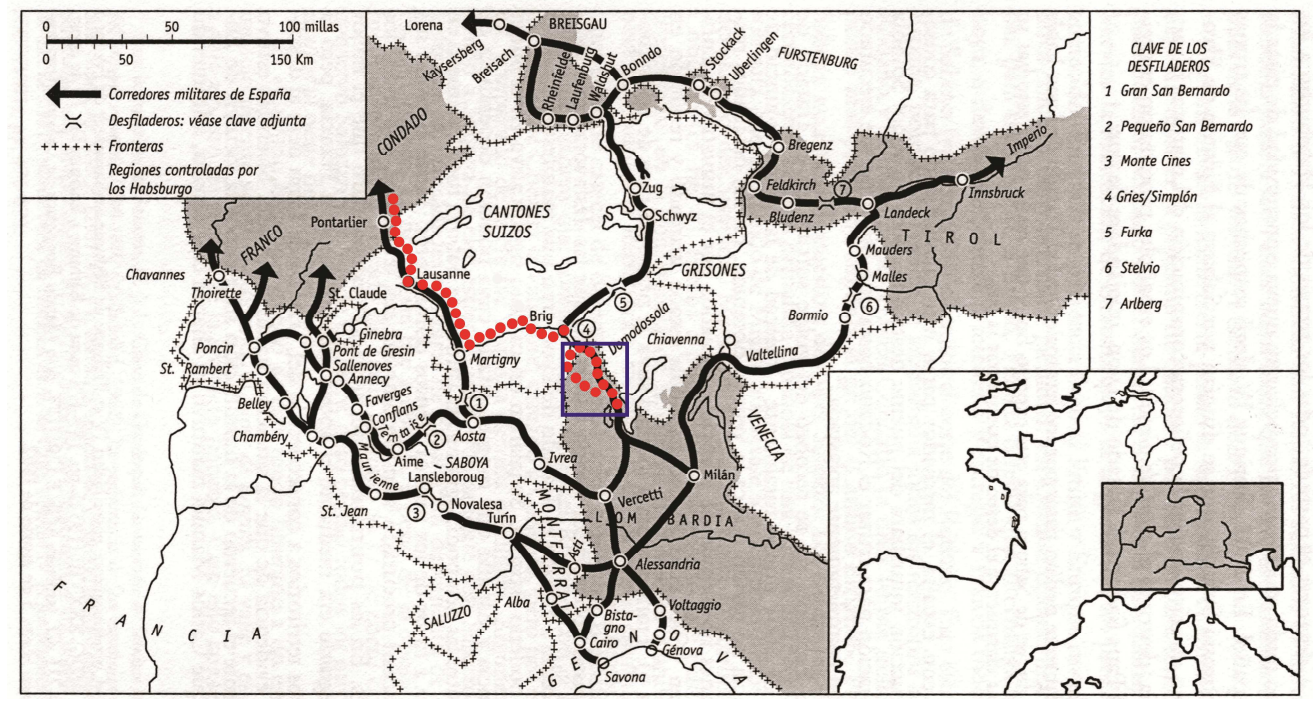

Fig. 4. La Lombardia: centro delle comunicazioni dell'Impero Spagnolo in Europa (Parker, 1985, p. 106), modificata dall'autore; tratto analizzato nel presente articolo (in rosso). Nel riquadro viola la zona della Val d'Ossola (vedere Fig. 5).

in epoca sforzesca alla fine del secolo $\mathrm{XV}$, ricalcando la caratteristica ancora medievale delle stesse (Fig. 1), la situazione rimase critica per tutto il corso dei secoli XVI e XVII con continue suppliche al governo spagnolo per le riparazioni necessarie al borgo causate dalle inondazioni del torrente Bogna e gli interventi da questo centellinati in base alle disponibilità finanziarie centrali (Negri, 2007; Negri, 2017). Solo dalla metà del secolo XVII, mutate le condizioni militari dei territori milanesi in mano spagnola per l'urto quasi inarrestabile delle forze francesi sul fronte occidentale, che misero in grande pericolo la cosiddetta "via spagnola" verso le Fiandre e che transitava anche per l'Ossola, si decise, a fine secolo, la trasformazione di Domodossola in cittadella fortificata alla moderna. I documenti relativi a questa mutazione sono conservati all'Archivio Storico Civico della Biblioteca Trivulziana di Milano. Per volere di Juan Tomás Enrìquez de Cabrera, Conte di Melgar e governatore dello Stato di Milano fino al 1686 e del suo successore Antonio Lopez de Ayala Velasco y Cardeñas conte di Fuensalida, il progetto delle fortificazioni alla moderna per la città fu affidato a Gaspare Beretta (Bossi, et al., 2007, pp. 4446) maestro di campo generale ed esperto inge- gnere maggiore dello Stato (Dameri, 2016), il quale lasciò una traccia scritta degli interventi necessari, insieme all'ingegnere Formenti, consistenti nella realizzazione di bastioni su ciascuno dei cinque angoli della cinta e profili di fortificazioni con spalti, strada coperta ${ }^{3}$ (Figg. 2 e 3) e fossato "alla moderna" insieme a dei pareri per la protezione della fortezza domese (Bossi, et al., 2007, p. 74). In effetti un progetto così grandioso aveva ben poche speranze di realizzazione pratica, anche per le continue e fastidiose inondazioni del torrente Bogna, ma i lavori iniziarono lo stesso, sul finire del secolo XVII, con la ricostruzione della torre distrutta dall'esplosione, sterri e profilature del terreno antistante il castello. Questi vennero però quasi subito interrotti sia per la mancanza dei necessari finanziamenti sia per la conclusione del dominio spagnolo in Lombardia a cui si sostituì quello austriaco.

\section{Il secolo XVII in Ossola}

La difesa dei territori del Ducato di Milano, costretto fra le mire espansionistiche veneziane a Est e sabaudo-francesi a ovest, nel corso del secolo XVII, fu affidata ad alcuni punti cardine, valutati in relazione all'importanza strategica del territorio difeso, llave de Italia e corazón de la 
monarquia posto sul camino español che conduceva alle Fiandre (Viganò, 2015; Scotti Tosini, 2003). Sino dalla fine del secolo XVI e per tutto il seguente, la descrizione cartografica del territorio, l'uso di planimetrie urbane organizzate in appositi atlas realizzati con intenzioni strategiche e propagandistiche (Dameri, 2013, 2014, 2015), l'attenzione allo stato delle fortificazioni urbane, ripetutamente oggetto di visite di ingegneri e la loro riconsiderazioni in funzione di anelli di una catena difensiva continua e collaborativa, riorganizzata in una nuova dimensione poliorcetica suggerita dall'esperienza fiamminga, caratterizzarono le intenzioni militari del governo spagnolo (Dameri, 2015, 2016, 2017, 2018). In modo particolare fu considerata decisiva la trasformazione delle cinte urbane in fase di degrado in fortificazioni bastionate alla moderna e a questo scopo i più importanti ingegneri militari, aggiornati ai più recenti trattati militari e formati alle nuove scuole di artiglieria, furono utilizzati in diverse campagne. Il cammino spagnolo verso le Fiandre, che dal Finalese ligure consentiva alle truppe di giungere a destinazione, transitava, a seconda delle alleanze politiche, attraverso il Franco Contado o i passaggi lacustri (Valchiavenna e Valtellina principalmente) compreso, marginalmente, anche il valico del Sempione verso la Svizzera (Fig. 4) (Parker, 1985, pp. 117-142). Anche l'Ossola fu quindi coinvolta nella difesa di questo itinerario, in linea di massima fornendo la protezione dell'attraversamento e gli alloggi necessari alle truppe, impegno gravoso chiesto agli ossolani probabilmente in cambio del mantenimento dei loro privilegi per l'esenzione dalle tasse. Tra le tante sciagure -la peste o le alluvioni del torrente Bogna- che gravavano sulla valle, la pretesa dei governanti spagnoli di sottoporre l'Ossola all'obbligo degli alloggiamenti degli eserciti in transito era particolarmente fastidiosa. Quando la guerra chiuse i valichi delle Alpi Occidentali, il "corridoio delle Fiandre", che collega le terre spagnole di Lombardia a quelle sul Mare del Nord, passò anche per il Sempione attraversando l'Ossola, protetta da un efficace sistema difensivo di torri, caseforti, castelli e chiuse alpine localizzate nei punti strategici chiave del percorso via- rio lungo il fiume Toce, proveniente dai laghi d'Orta e Maggiore (Fig. 5).

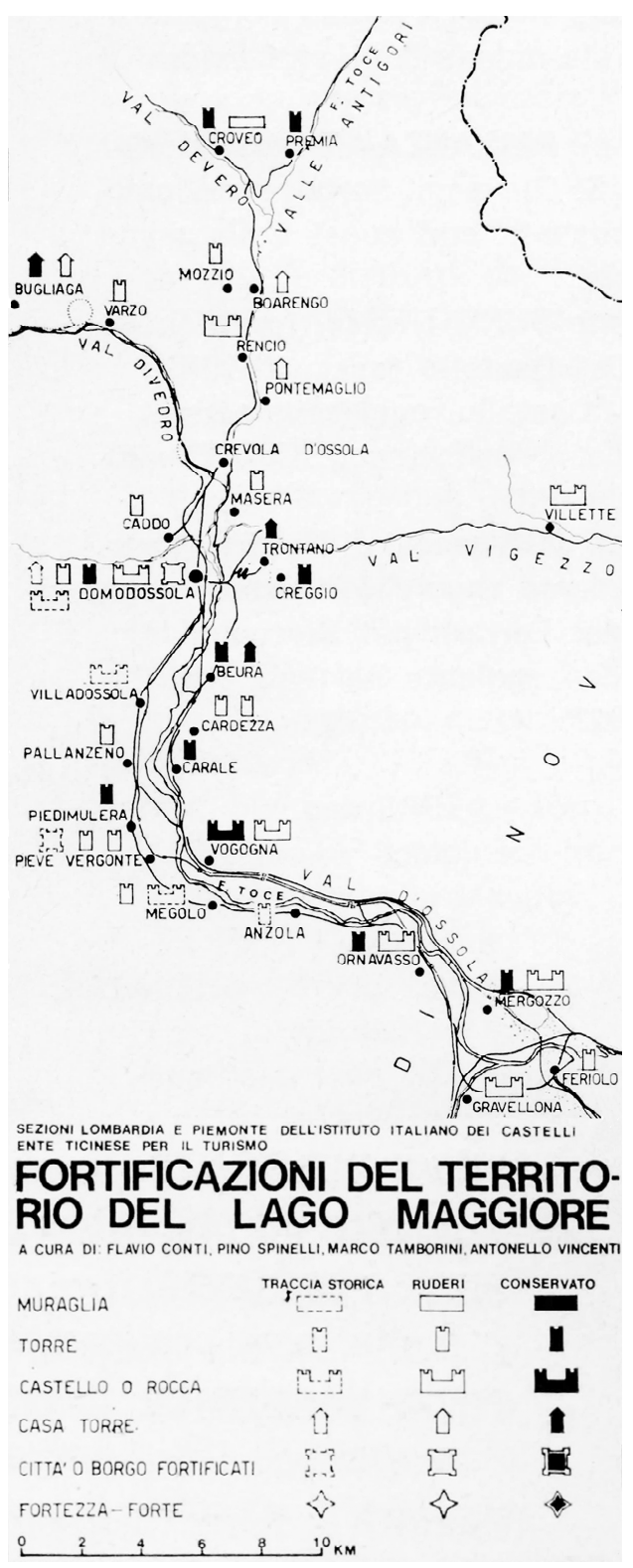

Fig. 5. Fortificazioni del territorio del Lago Maggiore.. Tratto da AA.VV. (1979) Fortilizi del bacino verbanese. Atti del Convegno sulle "Fortificazioni del territorio del Lago Maggiore"- Pallanza 1976, p. 115. Particolare della Valle dell'Ossola. 


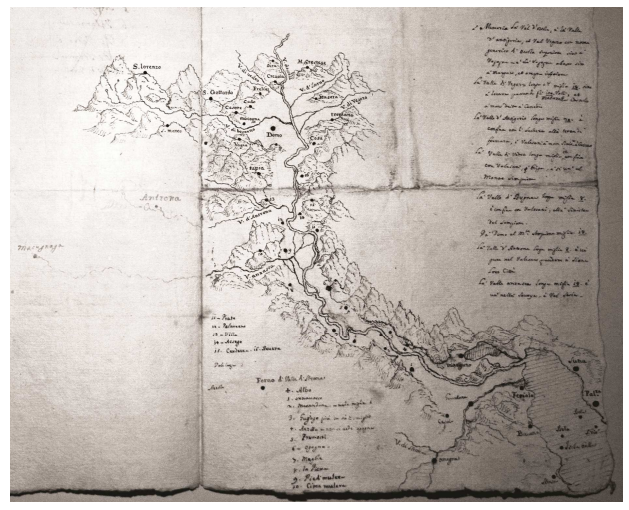

Fig. 6. BTMi, Belgioioso, 267, Vallese e Ossola "1674"- doc. 133. Anonimo, "Valle di Ossola", disegno a penna e matita, senza scala, 360 × $270 \mathrm{~mm}$, didascalie.

Se i traffici mercantili sul Sempione recarono beneficio al mercato di Domo, l'ininterrotto transito di militari mise in crisi l'economia della valle. Gli eserciti del re di Spagna, per muoversi attraverso l'Europa, dovettero poter disporre di vie sicure che consentissero il libero transito delle truppe.Le spese per l'esercito provocarono il crollo economico dello Stato, già gravato dalle carestie e dalle malversazioni di una burocrazia grigia e rapace. Così che imposero sempre nuove "contribuzioni", oltre all'obbligo di dare alloggio e pane ai soldati in transito, dovere al quale fu difficile per gli Ossolani sottrarsi. Il borgo di Domodossola tuttavia non fu mai oggetto di trasformazioni in cittadella bastionata almeno sino alla fine del secolo XVII. Non era necessario in quanto piuttosto isolata a nord e non minacciata da invasioni essendo protetta da una catena di montagne che non consentiva il trasporto dell'artiglieria pesante e non essendo mai stato minacciato da sud, protetto dalle fortificazioni lacustri di Arona. Per il governo spagnolo la "distanza" fu il vero enemigo público número uno, negli atti di governo per la trasmissione degli ordini, delle notizie e del materiale e per il movimento e lo spostamento delle truppe (Parker, 1985, p. 85). Solo un efficace sistema di posta, un organizzato sistema di trasporto di materiale e la protezione politica e militare delle truppe che transitavano sui "corridoi" europei potevano garantire la conservazione dei territori amministrati. Questo fu il motivo per cui anche in Osso- la, sull'antico esempio dell'Itinerario militare di Alberto da Vignate (1496-1519) conservato nella Biblioteca Braidense di Milano, nella seconda metà del secolo XVII, a fianco della idea di fortificare alla moderna il borgo domese, fiorirono una serie di campagne di rilievi manoscritti dei territori ossolani che, valle per valle, consentirono agli ingegneri da campo, arruolati nelle fila spagnole, di misurare e analizzare attentamente i percorsi e le distanze di percorrimento dei principali assi viari dell'intera vallata, annotandone i punti strategici e i passaggi tatticamente più utili alla difesa dalla temuta invasione da nord.

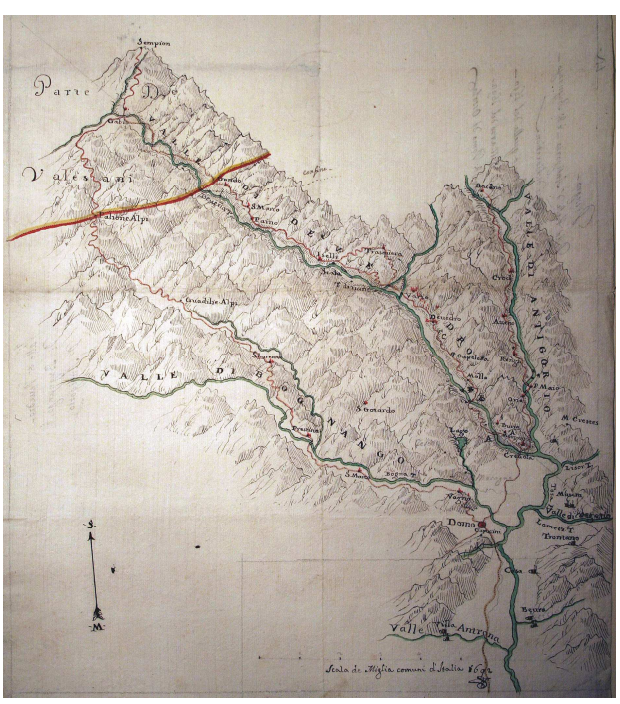

Fig. 7. BTMi, Belgioioso, 267, Vallese e Ossola "1692" - doc. 189 Anonimo, "Dissegno / Della Valle d'Antigorio - è di Bugnanco / con la sua dichiaratione / che è uniforme a quella del 1690 / l'ordine di S.E. di Marzo del 1692 / è nel piego di Domo de Ossola", disegno a penna a colori, mappa orientata, "Scala de Miglia comuni d'Italia 1692" (senza unità di misura), 298 x $416 \mathrm{~mm}$, didascalie, sul verso, "Valle di Deuedro / Di Bugnango, è / D'Antigorio".

\section{La cartografia manoscritta dell'Ossola e delle sue valli degli ingegneri da campo, della seconda metà del secolo XVII}

Presso 1'Archivio Storico Civico e Biblioteca Trivulziana di Milano, nel Fondo Belgioioso sono conservate le carte che documentano questo faticoso lavoro di rilievo del territorio che, a partire circa dal 1673, condusse alla realizzazione 


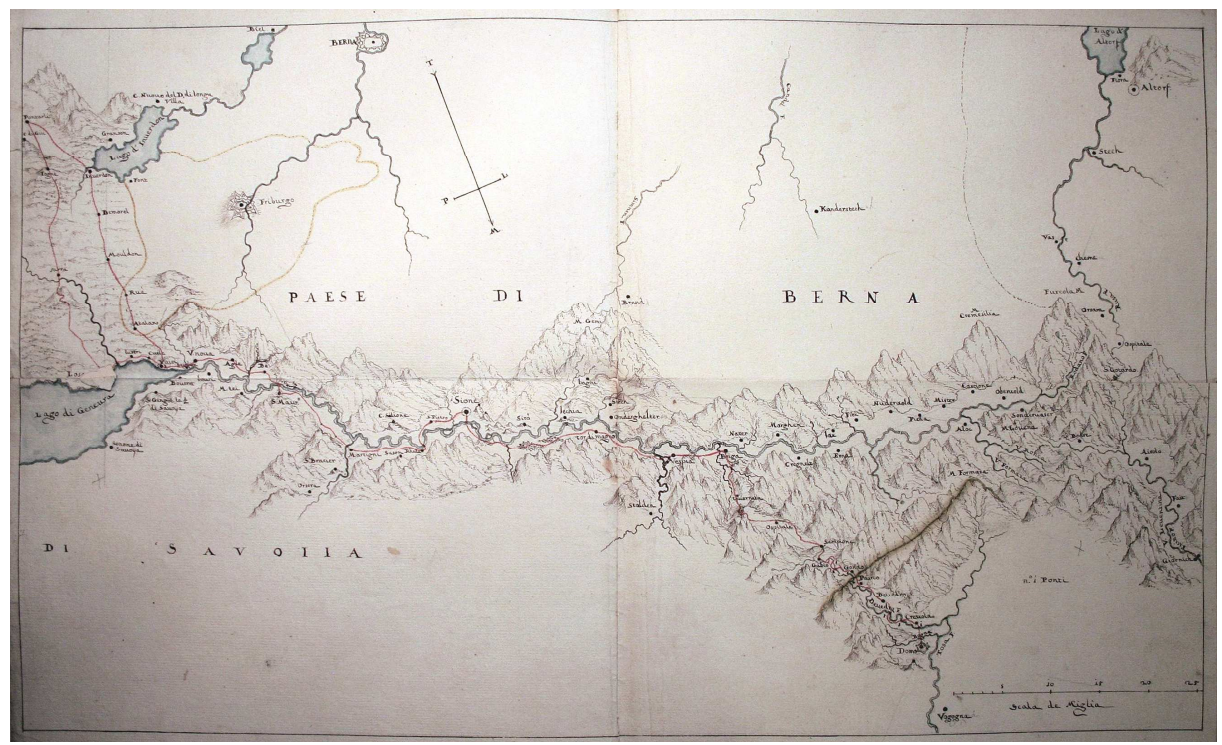

Fig. 8. BTMi, Belgioioso, 267, Vallese e Ossola "1674"- doc. 182 "Anonimo Paese di Berna" disegno a penna acquerellato, mappa orientata, paesaggi in rilievo, "Scala de Miglia 25", mm 585 x 358, didascalie, sul verso "Paese de Vallesani".

delle mappe manoscritte della valle dell'Ossola e afferenti, e parte anche del territorio svizzero di collegamento che da Briga porta verso la Francia via Losanna e Ginevra: la Val d'Antigorio, di Vegecio, di Canobio, Antrona, Valanzasca, di Vedro e di Strona ${ }^{4}$. Inoltre tre mappe più ad ampia scala, di notevole importanza, che conducevano dal lago Maggiore a Domodossola (Fig. 6), da Domodossola a Briga attraverso il passo del Sempione in due differenti vie, la Val Divedro o la Val Bognanco (Fig. 7) e tutto il percorso, in territorio svizzero che da Briga conduceva al Lago Lemano e oltre (Fig. 8). La cartografia manoscritta contiene i percorsi e i toponimi vallivi (alcuni scomparsi) con sommarie descrizioni paesaggistiche e sono accompagnate da relazioni descrittive che segnalano i punti di maggior interesse strategico utili a interrompere strade, fare crollare ponti o trincerare luoghi di difesa naturali. Quale esempio delle azioni da intraprendere per rallentare la discesa del nemico o impedire il trasporto di artiglieria pesante si propone una relazione sulle valli Divedro e Antigorio/Formazza del $1690^{5}$. Innanzitutto i ponti di legno, in caso d'invasione, dovevano essere distrutti: “[...] Primieramente il Ponte di legno di Creuola in occasione di sospetto si / deue distruuere -
[...]"; i gruppi di case si devono serrare in trincee: “[...] È le Case superiori dall'una all'altro serrarle con Trinchiera [...]"; le strade andavano interrotte o scarpate: "[...] Conuiene con le Stampe dè Minadori che sono qui senza perdita di / tempo prontarle in quatro [per rompere la strada], ò cinque parti, dal su.to Ponte di Creuola [...]”; “[...] E più oltre scarpare là strada con l'opera dè Guastadori- [...]"; era necessario "fornellare" i ponti: “ [...] dal su.to Ponte di Creuola / sino à q.lo di Vico come l'hò hà ueduto il Marchese Capo di q.ti Minadori / Vano fatti li Fornelli al su.to Ponte p[er] metterui la polvere al bisogno [...]"; "[...] Alli 23 - arriuai à Domo d'ossola doue ui trouai / il Conte Borromeo col quale nellà Valle Deuedro / sino al piede del Sempione si uisitò / et annotò il tutto [il giorno 24] anzi condussi meco il Marchese / Capo dè sei Minatori al quale feci uedere / ciò che doueua operare masseme al fornellare / li Ponti di nuouo, per metterui la polvere al / tempo preciso / et annotando anche le strade / da rompersi che propizi sono sul precipitio, è / facile (a mio credere) la sua difesa [...]"6; infine ripristinare le antiche fortificazioni già esistenti: "[...] Al Ponte di Pietra innanti un miglio chiamato il Passo stretto ui è una / Torre, è muraglia antichissima 
sin sotto là Montagna alla mano / dritta con suo Portone opera rimarcabile - A questa muraglia ui uà otturata l'apertura delli Merli, è farui / due Banconi di muro asciutto quali seruiranno [riempiti di terreno] di Terrapieno, et allà / Torre ui uà il suo coperto con bussola di Steccata [fodrato di Tauoloni], è Rastello, con sua ferram[enta] / douendossi occupare il sasso eminente che fa da fianco, è fornellare il Ponte -Et allà mano sinistra [del ponte sudetto], ui uà fatto altro [alto braza sette] muro, serrandossi sotto il gran sasso che / cade perpendicorarmente, e farui li suoi Banconi come già sopra, / allà sara molto salutare allà sudetta muraglia uecchia, et alla nuoua / farui per di fuori un Fosso tagliato nel sasso fondo l'altezza di un huomo / è largo altro tonto- Si deue pure fornellare il Ponte di Pietra chiamato del Golio".?

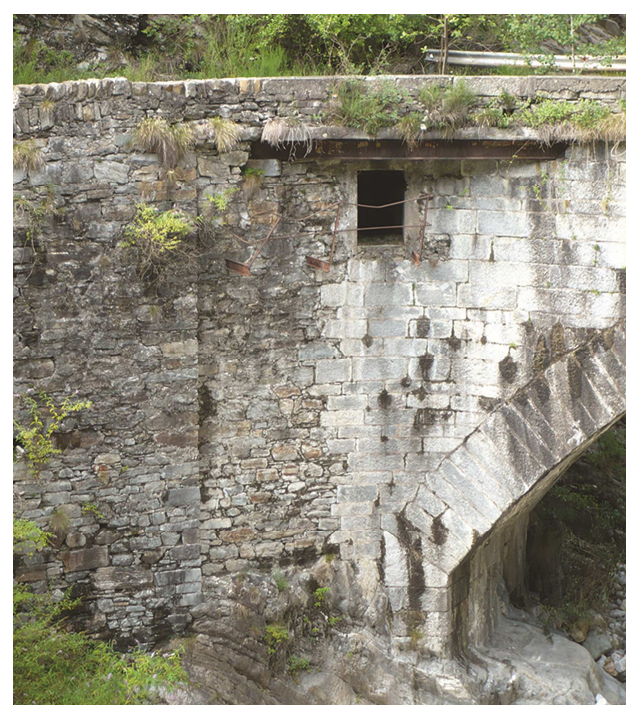

Fig. 9. Ponte con "fornello" in Valle Vigezzo (si ringrazia per la foto Pier Antonio Ragozza).

\section{Conclusioni}

La difesa dei territori spagnoli in Ossola non fu solo statica ma dinamica, rivolta alla conoscenza diretta del territorio e allo sfruttamento delle sue opportunità strategiche. La propensione a questo tipo di pensiero militare giunse fin'anche ai secoli successivi XVIII e XIX. Ancora oggi alcuni ponti in Ossola conservano ancora $\mathrm{i}$ segni dei "fornelli", anche se realizzati in epoche più tarde, come quello illustrato, in Valle Vigezzo (Fig. 9).

\section{Note}

${ }^{1}$ Una copia della relazione è oggi nell'archivio Enrico Bianchetti, da lui manoscritta, un'altra è conservata nell'Archivio di Stato di Torino (d'ora in poi ASTo), sez. Corte, 1768, provincia di Novara, fascicolo 10, Mazzo 70, Lettere diverse del marchese d'Ormea, Governatore di Novara, riguardanti l'escursione da lui atta nel Contado. (Rizzi, 1979).

2 ASTo, 1944, Paesi di nuovo acquisto, Fascicolo 25, Mazzo 69, Nota delli oggetti di Guerra estratti dai Forti di Arona e Domodossola come appare dagli originali sottoscritti dalli Guarda Magazzini e trasportati nel castello di Milano.

${ }^{3}$ Disegno allegato alla relazione: Vallese e Ossola "1690" - doc. 155-158: "li 6 - Decembre 1690 / Consegnata al Ayutante / Filippo And[re].a Celoti nella / Compagnia del S.r Conte / Carlo Borromeo Arese / B - A S.E. / Dandoli conto del mio operato dal giorno 18 nouembre che / partij dà Turino sino al sudetto 6. Decembre"; nel testo "[...] Li 30 - Visitassimo diligentemente Domo / è ui dissegnai una buona strada coperta, è disposi / il Terrapieno mentre con queste due operationi si uiene [ripetuto] si uiene ad'aprire il Fosso tutto cieco / è si scopre là Muraglia belissima, è fortissima / di pietra uiua [in Calcina] oltre l'accomodamento de muri, / Porte, è suoi picioli Reuellini, et il p.mo giorno di / Decembre hebbi terminato il tutto sul terreno [...]", "Arona li 6 / Decembre 1690", cc. 5 manoscritte, BTMi, Belgioioso, 267.

4 BTMi, Belgioioso cart. 267, sottocamicia "1674- / Valli di Ossola con altre che sboccano in essa / é di quella d'Auosta, docc. 130-142).

5 BTMi, Belgioioso, 267, Vallese e Ossola "1690"- doc 151. Anonimo, "All'Ecc[ellentissi] mo Sig.r Conte Carlo Borromeo / + / Memoria di quello si deue fare nelle Valli di Deuedro, di Antigorio, è di Formaza per la loro difesa in conformità delle Visite che si sono fatte", Domo d'ossola li 30 - nou[embr].e 1690, cc.2 manoscritte, Milano. 
${ }^{6}$ Quest'ultimo in BTMi, Belgioioso, 267, Vallese e Ossola "1690" - doc. 155-158. Anonimo. "li 6 - Decembre 1690 / Consegnata al Ayutante / Filippo And[re].a Celoti nella / Compagnia del S.r Conte / Carlo Borromeo Arese / B - A S.E. / Dandoli conto del mio operato dal giorno 18 nouembre che / partij dà Turino sino al sudetto
6. Decembre”, “Arona li 6 / Decembre 1690", cc. 5 manoscritto.

${ }^{7} \mathrm{Si}$ tratta dello sbarramento alpino di Croveo (Fig. 5).

Figure 1, 2, 3, 6, 7, 8 - Proprietà dell'Archivio Storico Civico Biblioteca Trivulziana; copyright @Comune di Milano - tutti i diritti di legge riservati.

\section{Bibliography}

AA.VV. (1979). Fortilizi del bacino verbanese. Atti del Convegno sulle "Fortificazioni del territorio del Lago Maggiore"-Pallanza 1976, p. 115.

Bossi, P.; Langè, S.; Rephisti, F. (2007). Ingegneri ducali e camerali nel ducato e nello stato di Milano (1450-1706). Dizionario bibliografico, Edifir, Firenze.

Bossi, P. (2017). "«Montaña con rios caudalosos a la frente, y lados, arroyos, fosos, bosque, lagos y fortalezas». Spunti per un aggiornamento delle conoscenze sul sistema difensivo dei laghi lombardi in epoca spagnola", in Echarri Iribarren, V. ed., FORTMED 2017. Defensive Architecture of the Mediterranean, XV to XVIII centuries, vol. V, pp. 311-318.

Dameri, A. (2013). Le città di carta. Disegni dal Kriegsarchivet di Stoccolma, Torino.

Dameri, A. (2014). "La notable campaña del año 1639 del marchese di Leganés. Disegni a Madrid e Stoccolma”, Lexicon, 19 , pp. 29-40.

Dameri, A. (2015). "Città sul confine: le guerre, la pace, le mura. Un atlante di disegni a Madrid", Studi Piemontesi, XLV, III, pp. 521-533.

Dameri, A. (2016a). "La difesa di un confine. Le città tra Piemonte e Lombardia nella prima metà del XVII secolo", in El dibujante ingeniero al servicio de la Monarquia hispánica. Siglos XVI-XVIII, Fundación Juanelo Turriano, pp. 271-293.

Dameri, A. (2016b). "«Servitore di due padroni». Gabrio Busca, ingegnere militare tra Piemonte e Spagna", in Defensive Architecture of the Mediterranean, XV to XVIII centuries, vol. II.

Dameri, A. (2016c). "Ingegneri in guerra. Pompeo Robutti e Gaspare Beretta al servizio della Spagna (1657)", in VI Convegno di Storia dell'Ingegneria, Napoli, pp. 627-634.

Dameri, A. (2017a). "Francesco Prestino and Giacomo Tensini, engineers at the service of the king of Spain. Fortifications reinforcement, city drawings", in FORTMED 2017. Defensive Architecture of the Mediterranean, XV to XVIII centuries, vol. V, pp. 159-166.

Dameri, A. (2017b). "Las «necesidades» de la guerra y historia de la ciudad. Los proyectos de los ingenieros militares en los archivos europeos", Utadeo, 5, pp. 130-141.

Dameri, A. (2018a). "Demolire per difendere. Lo smantellamento di fortezze nel XVII secolo", FORTMED 2018. Defensive Architecture of the Mediterranean, XV to XVIII centuries, vol. VII, pp. 87-94.

Dameri, A. (2018b). "«L'arte di pensare la guerra»: gli ingegneri militari e la costruzione della città", Atti e rassegna tecnica della Società degli ingegneri e degli architetti in Torino, 1, pp. 134-143.

Dameri, A.; Pozzati, A. (2018). "Tra Spagna e Austria: Giovanni Battista Sesti ingegnere militare", in FORTMED 2018. Defensive Architecture of the Mediterranean, XV to XVIII centuries, vol. VII, pp. 95-102.

Fior, M.; Viganò, M. (2004). "Architettura militare in Lombardia nei secoli XVII e XVIII", in Lombardia barocca e tardobarocca. Arte e architettura, Milano.

Negri, P. (2007). Un insolito riparo per il torrente Bogna: Domodossola, 11 febbraio 1624. Annales, Liceo Spezia, Domodossola, pp. 17-35.

Negri, P. (2014). "L'Albergo Terminus e d'Espagne di Domodossola; prime note storiche", Oscellana, 1, pp. 21-43.

Negri, P. (2017-2019). "Viatico per la storia del castello e delle fortificazioni di Domodossola", Oscellana, 2, pp. 81-106.

Parker, G. (1985). El ejército de Flandres y el Camino Español 1567-1659, Madrid.

Rizzi, E. (1979). "Immagini dell'Ossola nel '700", Oscellana, 3, pp. 113-117.

Rizzi, E. (2017). Domo borgo millenario. Storia di Domodossola dal X al XVIII secolo, Grossi, Domodossola.

Rotary Club Milano Aquileia. (1999). Città bastionate dello Stato di Milano, Milano.

Scotti Tosini, A. (2003). "Lo Stato di Milano", in Storia dell'architettura italiana. Il Seicento, Milano, vol. II, pp. 424-469.

Sesti, G.B. (1707). Piante della città, piazze e castelli fortificati in questo Stato di Milano con le loro dichiarazioni, Agnelli, Milano.

Viganò, M. (2015). “«Pax Hispanica»? Fortificazioni in lombardia nell'età di Filippo III (1598-1621)”, Quintana, 14, pp. 23-34. 